\title{
Correction to: Chemical-Mediated Alteration of Antibiotics
}

\section{Correction to: \\ Chapter 8 in: Sagar et al., Antibiotic Resistant Bacteria: A Challenge to Modern Medicine, https://doi.org/10.1007/978-981-13-9879-7_8}

In the original version of the book, the following corrections have been made:

The original version of the chapter 8, "Chemical-Mediated Alteration of Antibiotics," was inadvertently published without the references in the end.

The chapter has now been updated with the references at the end.

The updated online version of the chapter can be found at https://doi.org/10.1007/978-981-13-9879-7_8 